\title{
Architecting Methodology for Spatially and Temporally Distributed Resource Extraction Systems
}

\author{
Alessandro Aliakbargolkar ${ }^{1, ~}$ and Edward F. Crawley ${ }^{1,2}$ \\ ${ }^{1}$ Department of Aeronautics and Astronautics, Massachusetts Institute of Technology, Cambridge, MA 02139 \\ ${ }^{2}$ Engineering Systems Division, Massachusetts Institute of Technology, Cambridge, MA 02139
}

Received 15 January 2012; Revised 24 May 2012; Accepted 24 May 2012, after one or more revisions

Published online in Wiley Online Library (wileyonlinelibrary.com).

DOI 10.1002/sys.21230

\begin{abstract}
This paper presents a framework aimed at supporting the decision-making process for the preliminary design of system architectures for the extraction of spatially and temporally distributed resources. The goal of the research is to provide a tool to identify "global best" architectures for resource extraction "systems of systems," which usually differ from the sum of "local best" systems. The framework presented in this paper consists of an integrated model where both the architecture of the system of systems and the design of the individual systems are considered simultaneously. Using this approach, designers are able to gain insights by generating a selection of top-performing concepts. Subsequently, they can analyze those concepts using more sophisticated, albeit more resource-expensive design processes. The implementation of the proposed framework is a complementary tool to existing design practices. It features several advantages such as enabling the analysis of large-scale problems, for which exhaustive enumeration of architectures of systems of systems is not a viable option. The paper first presents an application of the methodology to the architecture of offshore oil and gas production fields. It then shows how to use the methodology for the development of scenario analyses. (C) 2012 Wiley Periodicals, Inc. Syst Eng 16
\end{abstract}

Key words: systems architecting; resource extraction systems; systems of systems

\section{INTRODUCTION}

Systems of systems require large capital investment for their development, and feature common challenges such as architectural complexity and plurality of feasible solutions. Multiple architectures can be designed for the extraction of resources, and very often the high number of feasible solu-

Contract grant sponsor: BP-MIT Major Projects Research Program.

* Author to whom all correspondence should be addressed (e-mail: golkar@skolkovotech.ru).

Systems Engineering

(C) 2012 Wiley Periodicals, Inc. tions prevents designers from considering all the available alternatives using traditional design methods. Comprehensive analysis of system architectures is hindered by complexity, due to emergent behavior resulting from the interaction of individual systems. For instance, feasible architectural solutions for an offshore oil platform depend on the design seawater depth, which is a function of the platform allocation on the offshore field. As a result of this coupling, system objectives that designers wish to pursue are oftentimes highly nonlinear. Henceforth, the definition of globally optimum system architectures is often a nontrivial exercise. Furthermore, system architects need to conduct several scenario analyses to identify the sensitivity to changing requirements and assumptions in architectures of interest for successive design iterations. Proper decision-making in preliminary stages of new projects 
is critical; in fact, as Saravi et al. [2008, p. 123] report: "70 to 80 percent of the life-cycle costs of a product are determined by decisions taken by designers during the early design stages" (of the design). If poor decision-making occurs at this stage, the value of the whole project can be significantly reduced.

Resource extraction is a problem encountered in several fields of engineering. An example from the energy domain is the architecture of an offshore field for oil and gas production, where system architects need to decide how many and which type of offshore facilities to develop, their allocation, and the temporal phasing or scheduling of tieback connections between reservoir wells and facilities over the project lifecycle. Similar issues are faced in aerospace engineering, such as in robotic exploration of the Solar System; consider, for instance, the architecture for a planetary surface exploration program, where architects need to decide the size and type of exploration systems to use (such as landers, rovers, or a combination of the two), plan their operations, and schedule their missions over time under budgetary and other constraints.

This paper provides a systematic approach for architecting resource extraction systems. It provides a decision-making support tool to analyze systems architectures under varying engineering and economics assumptions, resulting in a rapid assessment that complements traditional appraisal methods.

Maier [1998, p. 267] defines systems of systems as "systems that are built from components which are large-scale systems in their own right." Traditionally, the definition of an architecture for a system of systems is done heuristically through high fidelity expert assessments. This approach provides high accuracy in the evaluation, but constrains system architects to evaluate only one or two architectural concepts due to the high number of details to be considered in the assessments. As discussed by Prasad [1996], several organizations adopted concurrent engineering methods to improve the comprehensiveness of their assessments at a preliminary design stage; integrated development teams (IDTs) in concurrent engineering environments are able to develop and assess a handful of architectural concepts at a mid-fidelity level. In industrial practice, concurrent design represents the state of the art in preliminary design of system of systems. In academia, the design of system of systems has been approached by the emerging body of literature in systems architecting [Maier and Rechtin, 2002; Koo, 2005; Simmons, 2008; Hofstetter, 2009]. Quantitative system architecting employs low fidelity models to explore a large number of architectural concepts, therefore allowing the identification of promising concepts to be studied in more detail in successive design iterations. Agte et al. [2010] reviewed the use of multidisciplinary optimization frameworks for architectural optimization, focusing on optimizing architectures defined by expert assessments. Traditionally, optimization methods have been applied to parts of the problem, such as planning and scheduling (i.e., temporal allocation) [Arofnosky et al., 1962; Bohannon et al., 1970; Iyer et al., 1998; van den Heever et al., 2000] and spatial allocation [Devine et al., 1972; Starr, 1973; Barnes et al., 2005; Lin, 2008]. This paper draws from this literature, integrating quantitative systems architecting methods and heuristic optimization in a comprehensive systems architecting approach for the integrated problem, using low-fidelity models to explore a large number of concepts for systems of systems that are allocated in space and time, thus identifying a small set of preferred concepts to be analyzed with traditional mid-fidelity and high-fidelity expert assessments. Building on existing literature, the goal of this paper is to develop a comprehensive architecting approach for temporally and spatially distributed resource extraction systems and therefore allow the identification of promising architectural concepts to be studied in more detail with existing engineering design tools and methods. This goal is achieved by means of a low-fidelity architecture synthesizer that provides an unbiased assessment of concepts, evaluating them using system level metrics and providing flexibility to include additional evaluation metrics in the process.

The paper is structured as follows. Section 2 outlines the architecting approach as general methodology and defines the boundaries of the problem of interest. Section 3 applies the methodology to a case study for the architecture of offshore oil and gas production fields. Results are discussed to show how the architecting approach can be used for scenario analysis and identification of driving parameters within the architecture. Section 4 draws conclusions from the study and outlines avenues of future research.

\section{ARCHITECTING APPROACH}

This section describes the algorithmic approach proposed in this paper for the architecture definition of resource extraction systems. While the approach is general and can be applied to a variety of classes of systems of systems, Figure 1 shows the contextualization of the problem in the architecture of offshore oil and gas extraction systems. The figure shows the design variables that define the architecting problem in this specific domain, and identifies the five factors that characterize the architecture: the number of offshore systems, the architecture of individual systems (in the example, Floating Production, Storage, and Offloading vessels), the connectivity layout of systems with resources, and lastly the systems' spatial and temporal allocations (in the example, the allocation of offshore platforms and their production scheduling over time). The architecting approach is thereby generalized into five interconnected factors:

- Number: the determination of the number of resource extraction and processing system elements to be allocated. This task involves accounting for economies of scale and learning curves that affect the sizing of individual resource extraction systems.

- Resource Extraction System Architecture: the selection of promising architectural concepts for individual resource extraction system elements. This involves the description of architectural performance tradeoffs, cost, and risk of individual system elements and of the aggregate of the system elements as a whole.

- Connectivity: the determination of the optimal connectivity layout between resource extraction system elements and resources of interest. 


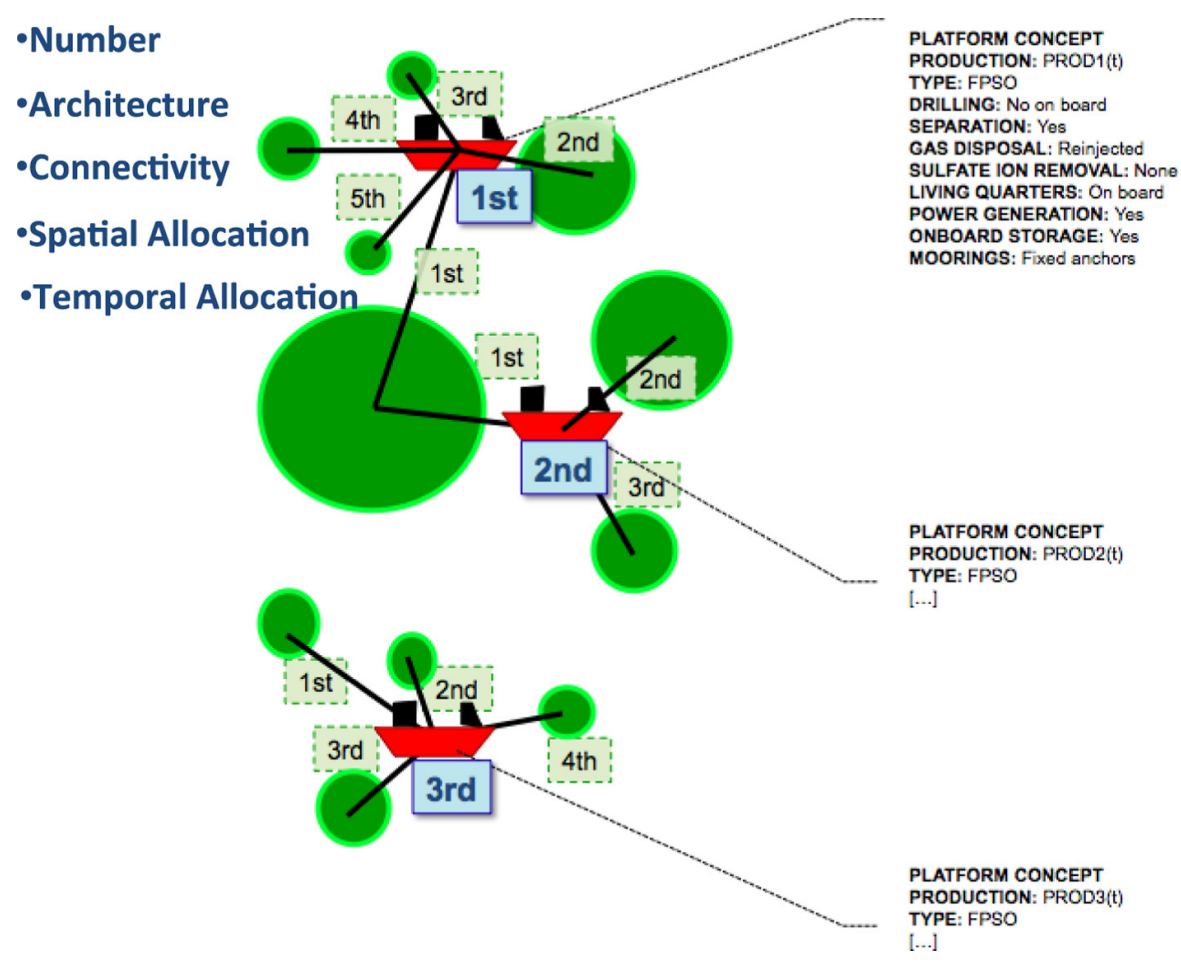

Figure 1. Offshore oil field architecting problem overview. [Color figure can be viewed in the online issue, which is available at wileyonlinelibrary.com.]

- Spatial Allocation: the determination of the spatial allocation of individual system elements to maximize the intended outcomes of the resulting system of systems.

- Temporal Allocation: the development of a strategy for temporal phasing of individual systems elements and their associated connection layouts over time.

The architecture of the corresponding system of systems is defined by the number of resource extraction system elements to be developed, their individual architecture, their connectivity scheme with the resources of interest, and their spatial and temporal allocation.

The approach is shown in Figure 2 with the steps described below. The approach consists of an algorithm featuring three iteration loops, called the inner iteration loop, the intermediate iteration loop, and the outer iteration loop. The inner iteration loop defines the optimal temporal allocation for connections for a single platform, the intermediate loop defines a feasible architecture, and the outer iteration loop restarts the algorithm to allow generation of multiple architectures in a Monte Carlo fashion. Randomness is introduced by Monte Carlo sampling in the selection of candidate extraction facilities. The algorithm ends when a predefined convergence criterion is met, such as when the algorithm consistently identifies a high level of similarity in top-ranking architectures. This criterion indicates convergence of the approach towards a globally optimal solution. Global optimality is estimated by comparing top architectures with an asymptotic optimum obtained by running the algorithm with no constraints. This is equivalent to comparing identified archi- tectures with a notional architecture where a single large platform extracts all resources in the shortest possible time. In the description that follows, inner loop iterations are indicated with a left superscript; intermediate loop iterations are indicated with a right subscript. Outer loop iterations are not shown for simplicity but are discussed at the end of the approach.

Step 1. Model input parameters. The model requires input parameters as preliminary assumptions characterizing the architecting model. Parameters can be used to describe environmental, economic, sociopolitical, and technical assumptions. The definition of model input parameters is a task to be accomplished by expert assessment and is part of the modeling problem for the specific application being sought. Table I provides examples of model input parameters for resource extraction systems.

Step 2. Develop a location grid for extraction facility placement. A location grid summarizes the geographical coordinates and other relevant parameters regarding the locations for potential placement of resource extraction systems. Examples of other relevant parameters for candidate locations are seawater depth in the offshore oil field example and terrain altitude in the planetary surface exploration example.

Step 3. Define/calculate connection costs between location grid and resources. Connections of different types must be established between resources and resource extraction systems. Connections may be physical (such as pipelines or electrical connections) or logical (such as possible traverses for a planetary exploration system). Costs are associated with the establishment of connections; these can be fixed costs (such as the installation of a pipeline), variable costs (such as 


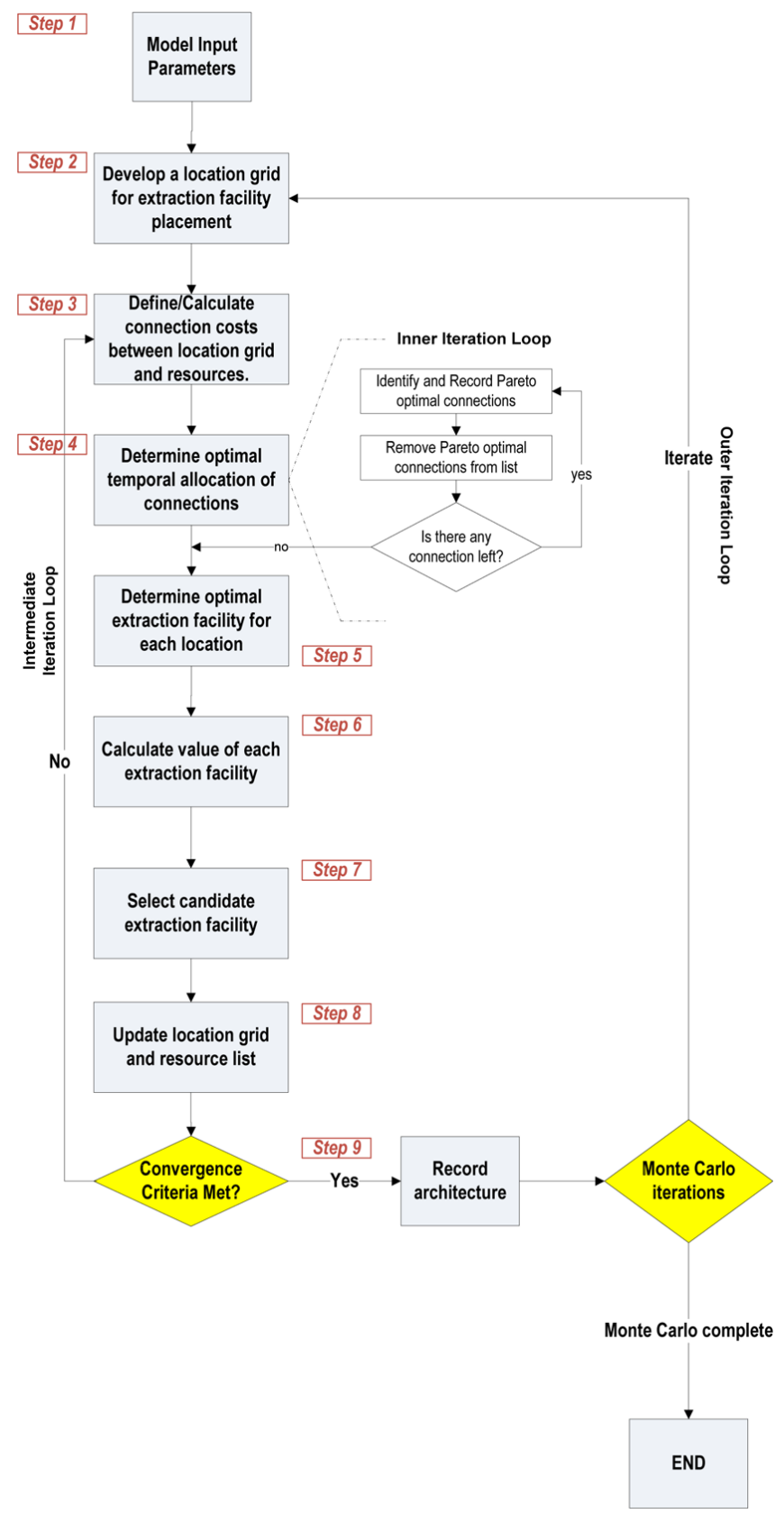

Figure 2. Proposed architecting approach. [Color figure can be viewed in the online issue, which is available at wileyonlinelibrary.com.]

the recurring cost for maintenance of a pipeline), or a combination of the two. Costs are either defined a priori by the system architect based on a set of heuristics, or they are calculated as a function of a set of proxy variables.

Step 4. Determine optimal temporal allocation of connections. Iterative Pareto screening can be used to determine an optimal temporal allocation of connections by defining an ordinal ranking of connection, thereby identifying an optimal resource extraction strategy over time in a relative ranking sense. This concept is explained by the following. For each
Table I. Examples of Model Input Parameters

\begin{tabular}{|l|l|l|}
\hline \multicolumn{1}{|c|}{ Assumption Type } & \multicolumn{1}{|c|}{ Offshore Oil Field } & \multicolumn{1}{|c|}{$\begin{array}{c}\text { Planetary Surface } \\
\text { Exploration }\end{array}$} \\
\hline Environmental & $\begin{array}{l}\text { Location grid, } \\
\text { Seawater depth profile. }\end{array}$ & $\begin{array}{l}\text { Gravitational } \\
\text { acceleration, Solar flux }\end{array}$ \\
\hline Economic & $\begin{array}{l}\text { Oil price, Well cost, } \\
\text { Well drilling cost, } \\
\text { Tieback cost per unit } \\
\text { length, operating cost } \\
\text { per unit production, } \\
\text { Discount Rate. }\end{array}$ & $\begin{array}{l}\text { Rover/Lander } \\
\text { development cost, } \\
\text { Launch vehicle cost }\end{array}$ \\
\hline Technical & $\begin{array}{l}\text { Project Lifetime, Well } \\
\text { Nominal Annual } \\
\text { Production Rate, } \\
\text { Maximum tieback } \\
\text { distance, Tieback } \\
\text { diameter, Maximum } \\
\text { tieback length, } \\
\text { Maximum number of } \\
\text { tiebacks per platform, } \\
\text { Maximum platform } \\
\text { production rate. }\end{array}$ & $\begin{array}{l}\text { Maximum roving } \\
\text { distance, specific } \\
\text { traverse energy density } \\
\text { (J/kg/km) }\end{array}$ \\
\hline
\end{tabular}

location $l \in\left[1,{ }^{1} N_{L}\right]$ in the grid (where ${ }^{1} N_{L}$ is the set of available locations at iteration 1), possible connections are characterized by cost and resource value. Let ${ }_{l}^{1} \vec{J}_{1}=\left[\begin{array}{ll}{ }_{l} C_{1} & { }_{l} V_{1}\end{array}\right]^{T}$ and ${ }_{l} \vec{J}_{2}=\left[{ }_{l} C_{2}{ }_{l} V_{2}\right]^{T}$ be the objective vectors representing the $\operatorname{cost}{ }_{l} C_{i}$ and value ${ }_{l} V_{i}$, where $i=1,2$ are two possible connections to resources from location $l$. Assuming objective maximization is desired, connection $1 \mathrm{domi}$ nates connection 2 if ${ }_{l}^{1} \vec{J}_{1}>{ }_{l}^{1} \vec{J}_{2}$. Iterating this procedure for all connections will result in the identification of a first Pareto front consisting of Pareto optimal connections for location $l$ at iteration 1, which are the first connections to be pursued as they feature the best value-cost tradeoff in the connection list. Removing such connections from the list and iterating, a second Pareto front can be obtained, and so forth, until all connections are removed from the connection list. This criterion provides a scheduling between subsets of connections. A finer scheduling across subsets is achieved by using a heuristic criterion. For instance, one might be interested in pursuing first connections tapping to resources with higher values, or connections featuring lower costs. Other heuristic criteria can be used or one could employ more sophisticated analysis such as Multi Attribute Utility Theory [Keeny and Raiffa, 1976]. Iterative Pareto screening is done across all available locations, yielding to a set of potential extraction facility allocations. The following step before making any allocation selection is to determine the optimal facility to be developed for each location.

Step 5. Determine optimal extraction facility for each location. The selection of the optimal architecture for an extraction facility in a given location can be framed as a decision-based systems architecting problem [Simmons, 2008]. This can be conceptualized in five substeps:

(a) Identification of architectural elements, that is, the formal decomposition of the system into subsystems, or simpler aggregates.

(b) Enumeration of feasible extraction facilities, based on a function-form decomposition and the definition of a 
structural morphological matrix such as described in the architecting framework by Simmons [2008]. Architectures are enumerated by selecting one possible choice for each formal attribute, while respecting a set of constraints. Constraints ensure the avoidance of architectures with no physical or logical meaning, such as placing a terrestrial-based extraction system in an aquatic environment.

(c) Development of a set of metrics to evaluate the architectures enumerated in point 2 . This can be done using parametric relationships to evaluate metrics of interest with respect to primary system drivers.

(d) Evaluation of architectures with the metrics developed in point 3 . Since multiple metrics are involved, the evaluation is a multiple-criteria decision process [Zeleny, 1982]. Such evaluation leads to the identification of Pareto-optimal architectures.

(e) Ranking of architectures must be performed in order to select the platform design concept to be used in the offshore field architecture. Ranking can be performed using Multiattribute Utility Theory [Keeney and Raiffa, 1976], assigning weights to different metrics or key performance indicators and thereby determine the ranking of Pareto optimal architectures.

Step 6. Calculate value of each extraction facility. The value of each extraction facility is defined by the difference of the total value of the associated resources and the costs associated with facility and connection operations. If value and costs are not expressed in the same units, an exchange rate must be agreed upon by decision-makers to proceed with the evaluation. For example, in the offshore field problem the exchange rate can be represented by oil price, which converts barrels of oil (a measure of resource value) into dollars.

Step 7. Choose candidate extraction facility. Once value is assessed for each potential extraction facility, a candidate facility is selected, and the definition of the whole architecture proceeds. Due to potential nonlinearities and the presence of local optima, the selection of the "best" facility-connections assignment does not always correspond with the "best" global architecture when multiple facilities are required. For this reason, the proposed approach implements a broader exploration of the design space; at this point, a random selection of the candidate extraction facility and associated connections is made. This decision is recorded, and the algorithm proceeds evaluating whether to proceed to a further run of the intermediate iteration loop or stop.

Step 8. Update location grid and resource list. The selection of a candidate extraction facility represents an assignment of a facility to a location and to a set of resources by means of connections. The location grid and resource list must be updated to reflect this assignment, by recording this choice and removing the assigned location and assigned resources from further iterations of the algorithm.

Step 9. Convergence criteria. Convergence criteria stop the intermediate loop and outer loop of the algorithm. These condition are defined arbitrarily by decision-makers; a possible condition consists in running iterations until all extraction facilities with positive value have been identified. Other possible conditions include stability of results and maximum number of iterations. The selection of convergence criteria is a function of the specific problem of interest.

\section{APPLICATION CASE STUDY: OFFSHORE OILFIELD ARCHITECTURE}

Section 2 discussed the proposed approach for systems architecting of resource extraction systems. This section contexualizes the approach to an application case study of offshore oilfield architectures. Section 3.1 provides domainspecific required background to frame the problem. Section 3.2 describes the application of the approach to the case study; Section 3.3 discusses results obtained in the application and comments on the examples of the scenario analyses. Interested readers can refer to Leffler, Pattarozzi, and Sterling [2003] and Raymond and Leffler [2006] for additional background information on offshore production systems.

\subsection{Background}

Offshore oil and gas developments occur in marine hydrocarbon basins with significant concentrations of commercially viable oil and gas reservoirs. After identification of potential hydrocarbon traps through seismic survey the exploration companies will drill wells to access and prove existence of hydrocarbon resources. This is followed by further seismic studies and use of appraisal wells to sample each reservoir and confirm the scale of the recoverable hydrocarbon reserves. This allows companies to confirm commercial viability. It is at this stage that the search for the optimal development configuration is pursued. This leads to the creation of a development plan and an execution project to create the infrastructure. Where the hydrocarbon resource is highly geospatially distributed, such as multiple smaller reservoirs in deep water, subsea "wet tree" wells are often used in place of or in addition to platform drilled "dry tree" wells. Subsea wells are connected to the production platforms through manifolds and flowlines referred to as tiebacks, which allow untreated reservoir fluids to flow from the subsea wells to processing platforms. The maximum length of flowlines is often determined by physical constraints, such as the reservoir energy to maintain pressure or the ability to avoid hydrate formation and blocking of flowlines on cool down when wells are shut in (i.e., end of production). Reservoir fluids flow through flowlines driven by the pressure in the reservoir. However, as soon as production begins reservoir pressure will decline unless there is a natural reservoir drive source or water and other injection techniques used to replace the hydrocarbon volumes to maintain reservoir pressure.

Detailed production profile simulations require modeling reservoir fluid physics using mass conservation equations and other reservoir dynamics. A simplified approach can be employed for preliminary architecture screening purposes, allowing the evaluation of a high number of simulations while being able to identify promising architectures at a high level of abstraction.

The simplified model represents a production profile as a function of time, as defined in Figure 3: 


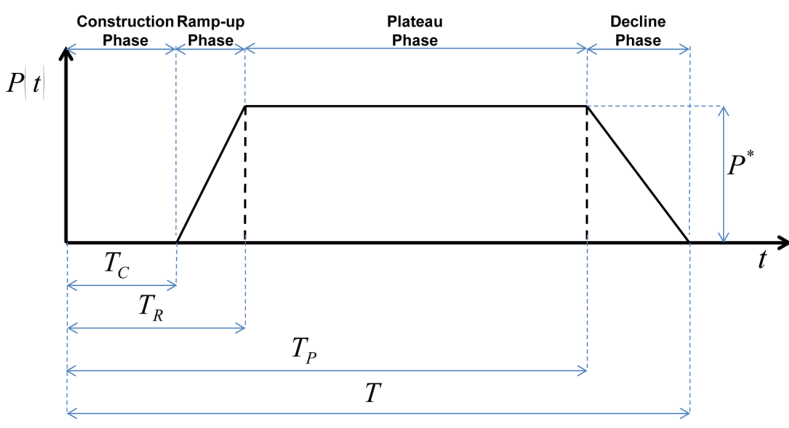

Figure 3. Production profile. [Color figure can be viewed in the online issue, which is available at wileyonlinelibrary.com.]

$$
P=P\left(t, T_{t}, T_{R}, T_{P}, T, P^{*}\right)
$$

Development concepts are evaluated accordingly to a set of user-defined performance metrics, i.e., a set of proxy evaluations for profit, technical risk, operational risk, safety, carbon emissions, and so forth. For instance, profit is often evaluated with an estimate of the Net Present Value (NPV) generated by the offshore development. This is done through the simulation of a production profile as a revenue stream and the phased capital and operational cost expenditure. These are combined to calculate the associated net cash flows and the net present value of development.

\subsection{Architecting Approach Implemented}

Given domain-specific background, the architecting approach can be implemented. In this application case study, the field architecture refers to the technical plan for the development, including information on the numbers of system elements, spatial allocation, facilities design, connectivity, and temporal phasing. A location is a geographical point on the field on which it is possible to locate an offshore facility (platform or manifold). Every location is characterized by its own geographical coordinates $\left(x_{i}, y_{i}\right)$. A location grid is a set of locations on which to conduct the architectural screening for field architectures. A tieback is a platform-production well connection (including flowlines and manifold). Tieback capacity refers to the potential hydrocarbons associated with the reservoir(s) to which the tieback is connected. Tieback distance is the tieback length between the platform location and the furthest well.

Step 1. Model Input Parameters. Model input parameters for the case study are presented in Table II. Input parameters represent the information required to characterize the available resources for an offshore project and preliminary assumptions set by decision makers.

Step 2. Develop a location grid for facility placement. A regular spaced grid of locations over the area of interest is considered in this case. This grid selection can be refined in further iterations, removing noninteresting locations and focusing the analysis on the locations included in best ranking architectures.

Step 3. Define/calculate connection costs between location grid and resources. Connection cost is assumed to be
Table II. Offshore Oil Field Architecting Model Parameters

\begin{tabular}{|l|c|c|}
\hline \multicolumn{1}{|c|}{ Parameter } & Units & Allowable Range \\
\hline Location grid & - & - \\
\hline Seawater depth profile & $\mathrm{ft}$ & - \\
\hline Project Lifetime & Years & $10-30$ \\
\hline Oil price & $\$ / \mathrm{bbl}$ & $(0, \mathrm{Inf})$ \\
\hline Well cost & $\$ \mathrm{M} / \mathrm{well}$ & $(0, \mathrm{Inf})$ \\
\hline Well Nominal Annual Production Rate & $\mathrm{Kbbl} / \mathrm{year} /$ well & $(0, \mathrm{Inf})$ \\
\hline Tieback cost per unit length & $\$ / \mathrm{km}$ & $(0, \mathrm{Inf})$ \\
\hline Operating cost per unit production & $\$ / \mathrm{kbbl}$ & $(0, \mathrm{Inf})$ \\
\hline Discount Rate & $\%$ & {$[0 \%, 100 \%]$} \\
\hline Maximum tieback distance & $\mathrm{km}$ & $(5,50)$ \\
\hline Tieback diameter & $\mathrm{m}$ & $(1,10)$ \\
\hline Maximum tieback length & $\mathrm{Km}$ & 40 \\
\hline $\begin{array}{l}\text { Maximum number of tiebacks per } \\
\text { platform }\end{array}$ & - & 10 \\
\hline Maximum platform production rate & $\mathrm{Kbbl} / \mathrm{day}$ & 50 \\
\hline
\end{tabular}

proportional to the Euclidean distance to be covered by the tieback. The distance between grid points and reservoirs is recorded in a distance matrix $D=\left\{d_{i j}\right\}$, where the $d_{i j}$ element records the distance between points $i$ and $j$. The "tieback cost per unit length" parameter serves as an exchange rate to convert distance into dollar numbers. Furthermore, a maximum distance constraint is enforced between platforms and reservoirs. If the maximum tieback length is defined as $d_{\max }$, reservoir $j$ is reachable from location $i$ if the following inequality constraint is met:

$$
d_{i j} \leq d_{\max }
$$

Step 4. Determine optimal temporal allocation of connections. Tiebacks are prioritized according to a tradeoff on proxy variables for cost and value. Tieback cost is proportional to the distance to be covered, whereas value is proportional to the total recoverable resources in the reservoirs accessed (i.e., reservoir capacity). Feasible tiebacks are plotted in a distance-capacity space; Figure 4 shows this plot for

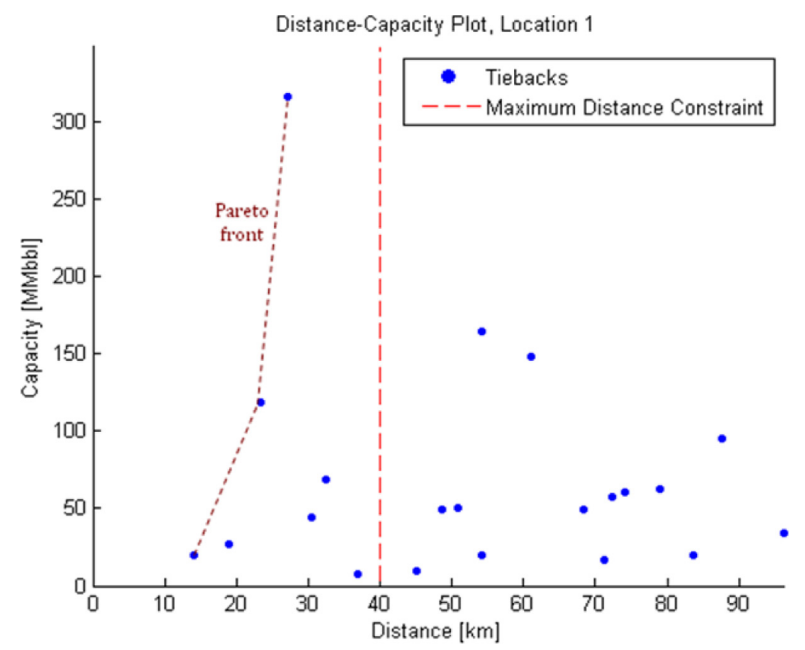

Figure 4. Distance-capacity plot, location 1, Pareto front. [Color figure can be viewed in the online issue, which is available at wileyonlinelibrary.com.] 
location grid point 1 (location 1) for the case study analyzed in this paper. The blue dots represent possible tiebacks as seen from location 1 . The vertical dashed line represents the maximum distance constraints as discussed previously. Therefore, the tiebacks lying on the left of this line are feasible, and the tiebacks lying on the right are infeasible. Iterative Pareto screening determines optimal temporal allocation, where the variables of interest are tieback distance and reservoir capacity. Tiebacks are prioritized according to their associated capacity. Dominated tiebacks (i.e., tiebacks that are not on the Pareto front) are deferred to successive platform developments until maximum platform capacity is achieved. Figure 5 shows the complete sequencing for the example presented in this paper.

Step 5. Determine optimal processing platform for each location. This step requires the estimate of the maximum on-site production capacity. The optimum tieback selection for each field location is reached when the corresponding assigned offshore processing platform achieves its maximum capacity. In most cases it is not possible to produce all the tiebacks at their maximum reservoir potential. This is due to the upper constraint given by the maximum platform capacity and production may have to be deferred. If a platform is already committed to produce $p$ resources and $p^{*}$ is the maximum allowable production capacity, an additional tieback is produced at most at a production rate of $\left(p^{*}-p\right)$.

The five substeps are implemented as follows:

(1) Identification of architectural elements: Offshore platforms are decomposed into topsides (equipment for oil and gas processing, utilities, manning, storage and offloading), substructure (underlying structure/hull) and foundations (piles or moorings to the seabed).

(2) Enumeration of feasible extraction facilities: Platform concepts are enumerated by full enumeration. The discussion of architectural enumeration is outside the scope of this paper; two extensive reviews of this topic

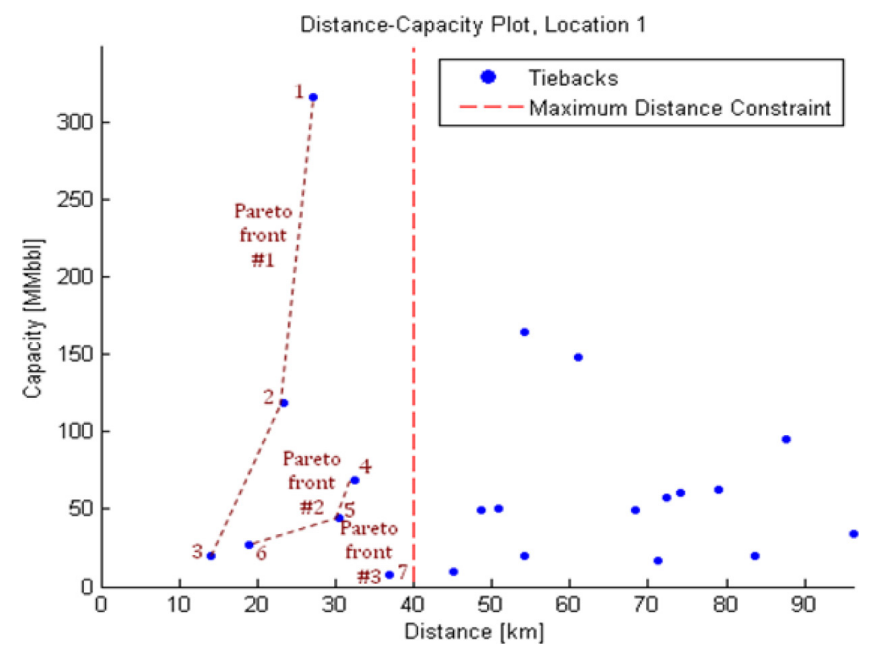

Figure 5. Distance-capacity plot, location 1, complete Pareto sequencing example. [Color figure can be viewed in the online issue, which is available at wileyonlinelibrary.com.] can be found in Koo [2005] and Simmons [2008]. The enumeration is regulated by a set of constraints, which ensure that nonsensical concepts are avoided. A nonsensical concept is one where the technology elements would not be logically combined such as a floating, Production, Storage, and Offloading hull with steel foundation piles (i.e., a compliant floating platform employing steel foundation piles, which are only used on rigid fixed offshore platforms only).

(3) Development of a set of metrics to evaluate architectures: This can be done using parametric relationships, as discussed previously. For example, fixed cost for an offshore platform can be estimated in first approximation as a function of concept elements, crude production rate, and seawater depth. Technical risk can be assessed with the development of a Technology Readiness Level (TRL) scale, a semiqualitative measure of the technical maturity of architectural concepts.

(4) Evaluation of architectures: Capital Expenditure (Capex) and Total Technology Readiness Level (TRL) can be used as metrics to identified nondominated platform architectures.

(5) Ranking of architectures: A criterion for ranking development architectures is required to pick a "best performing" solution. As discussed previously, such criterion is defined by decision-makers, and can be based on either a formal evaluation method (such as Multi Attribute Utility Theory [Keeney and Raiffa, 1976]) or based on decision-makers preferred heuristics, for example, by selecting the architecture with the lowest TRL on the Pareto front for each location in the grid (see Figure 6).

Step 6. Calculate value of each development option. The value of development options is defined by the Net Present Value (NPV) of expected profit, defined as the value in today's money (allowing for discount rate) of the revenue associated with oil production, less the costs associated with the development installation and operations.

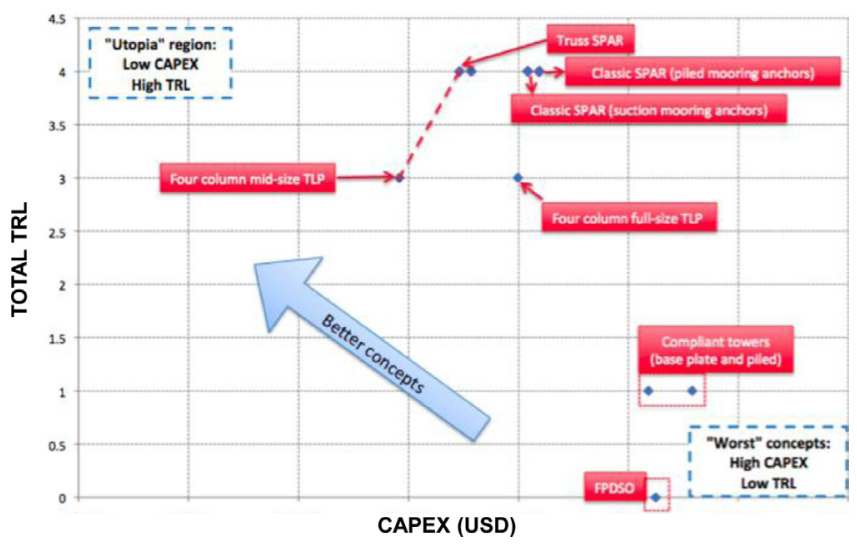

Figure 6. Example of platform architecture evaluation. [Color figure can be viewed in the online issue, which is available at wileyonlinelibrary.com.] 
Step 7. Select candidate development option. The development option selection for the architecture considered is generated randomly, as discussed previously.

Step 8. Update location grid and resource list. Tiebacks associated with the selected platforms represent a commitment of reservoir resources. The reservoir list is updated by removing the resources that have already been allocated. The location grid is updated removing used platform locations from the location grid.

Step 9. Convergence criteria. Convergence is met when either there are no more reservoirs to be allocated, or when no further candidate development option with positive value can be found.

\subsection{Offshore Field Architecture: Results}

This section discusses the results obtained applying the architecting approach to a notional case study in offshore oilfield architecting.

Figure 7 shows the results in terms of architectural ranking by Net Present Value of Profit (NPV). Architectures of interest are the ones in the top left corner of the graph. It can be seen that, in proximity of the top-ranking architecture, the graph tends to be convex. This is an indicator that optimality has been reached, since dominant schema emerged and therefore top ranking architectures feature a high degree of commonality.

Figure 8 shows the top-performing architecture for the baseline input model parameters considered. This result is function of the input parameters provided to the model; a different answer would result if parameters were changed. The proposed architecting model provides flexibility to change parameters and therefore conduct scenario analyses, by changing parameters and assessing their impact on the overall architecture. Consider the case in which system architects want to assess the impact of revenue per unit production in their project. If we assume that the oil price increases by a factor of 2.5, we can assess the impact of this parameter in NPV of profit. As shown in Figure 9, the maximum NPV is now $\sim 61 \$ B$, which represents therefore an increase in profit by a factor of 3.4. Such increase is not linear with the increase

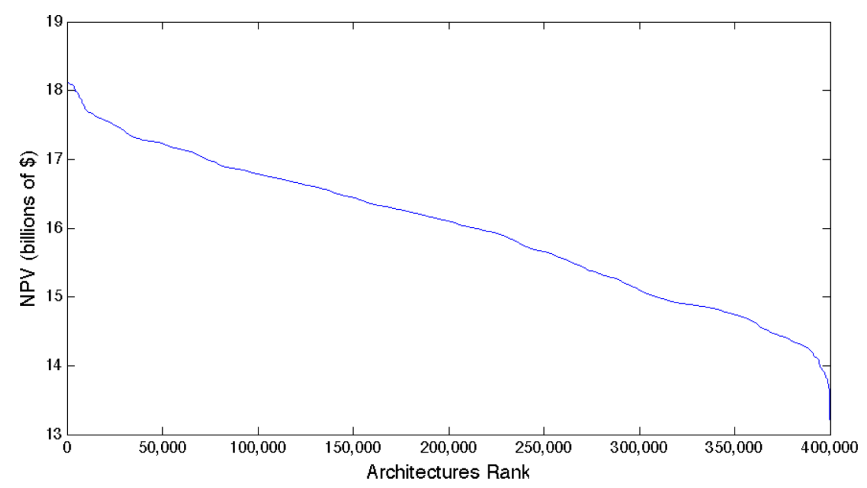

Figure 7. Baseline case, architectural ranking by profit. [Color figure can be viewed in the online issue, which is available at wileyonlinelibrary.com.]

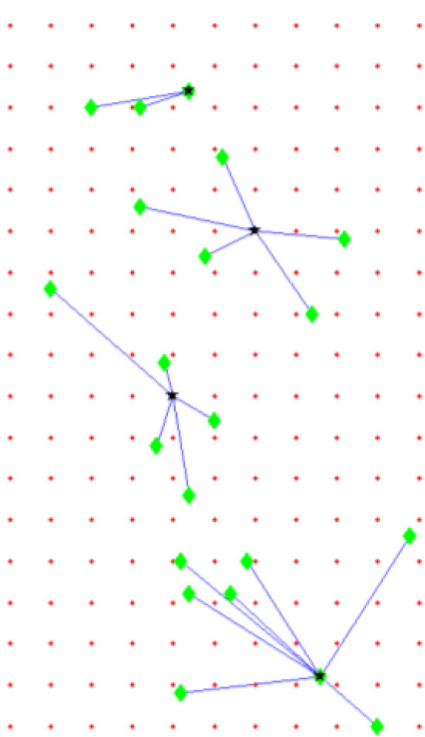

Figure 8. Baseline analysis, top four architectures. [Color figure can be viewed in the online issue, which is available at wileyonlinelibrary.com.]

in revenue due to the nonlinearity and couplings that exist in the architecting problem.

Another demonstration of coupling effects comes from the following scenario analysis. Consider the case where the maximum production rate per offshore platform is halved. The main result expected from this parametric change is that more platforms are required to produce the entire field, since the overall capacity of the reservoirs is held constant. This translates into pipeline networks covering smaller distances, since the architecture is more geographically distributed. Since capital cost associated with platforms dominates over the capital cost associated with tiebacks in the example considered, the overall NPV is reduced, as shown in Figure 10. Figure 11 shows the best field architecture in this case.

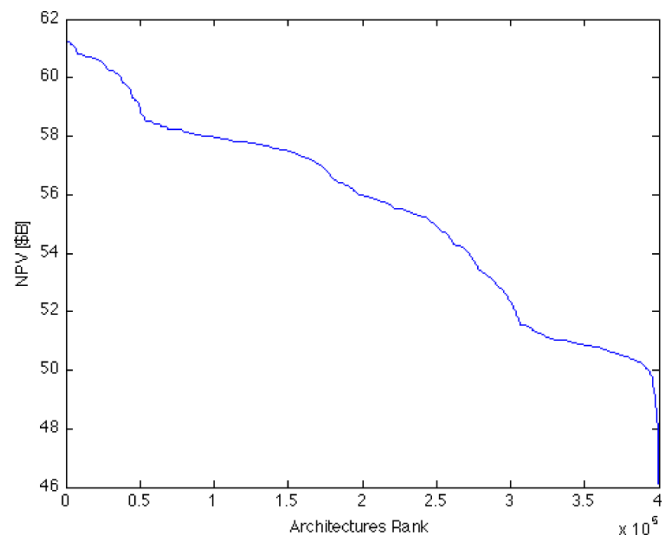

Figure 9. 2.5 $\times$ increase in oil price, architectural ranking by profit. [Color figure can be viewed in the online issue, which is available at wileyonlinelibrary.com.] 


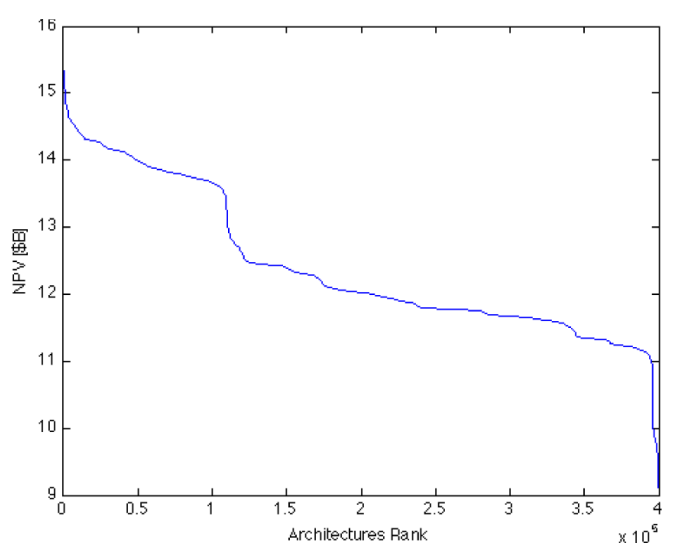

Figure 10. Halved production rate, architectural ranking. [Color figure can be viewed in the online issue, which is available at wileyonlinelibrary.com.]

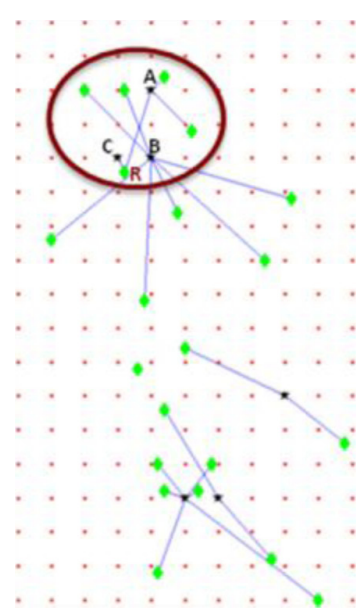

Figure 11. Example of crossing resulting from coupling in the offshore architecture example. [Color figure can be viewed in the online issue, which is available at wileyonlinelibrary.com.]

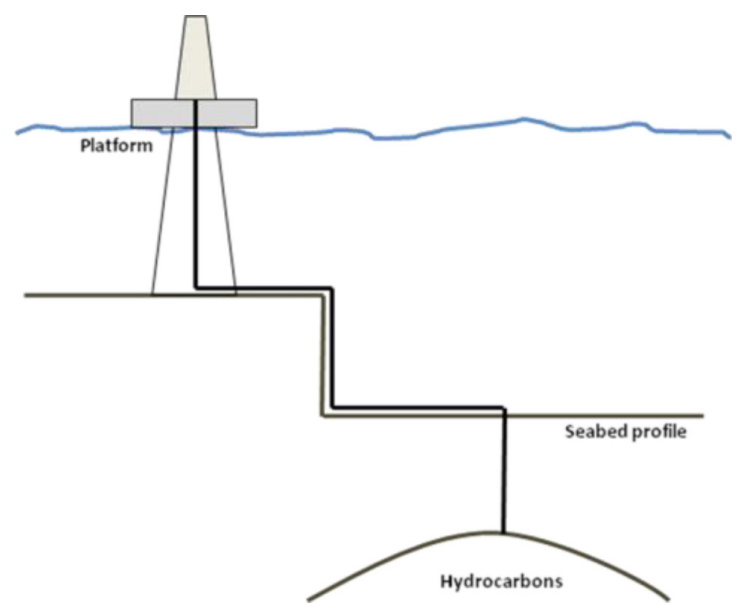

Figure 12. Platform positioning notional example. [Color figure can be viewed in the online issue, which is available at wileyonlinelibrary.com.]
This scenario also shows an example of coupling in the architecture. In this example, reservoir $\mathrm{R}$ is produced by platforms $\mathrm{A}$ and $\mathrm{C}$. When maximum production rate is reached by the closest platform (platform A), any remaining resource in the reservoir is produced by nearby facilities with spare production capacity (platform B). Platform C is slightly displaced from the reservoir, because the water depth there is shallower (as shown in Fig. 12). Such positioning increases connection cost, but reduces the overall field architecture cost, hence increasing profit. This result is an example of the importance of coupling the design of individual offshore processing platform to the overall field architecture.

\section{CONCLUSIONS}

This paper presented a unified framework for architecting spatially and temporally distributed resource extraction systems. The proposed low-fidelity approach is suited for preliminary studies of new development projects, highlighting architectural tradeoffs that emerge from the characteristics of the specific project considered. This paper provides a general approach that can be tailored to applications in multiple disciplines, and provides a case study in offshore oil field development planning to show the implementation of the general methodology with an example. The example also shows the importance of conducting scenario analyses, and the relevance of coupling the architectural analysis of individual systems to their performance as part of a system of systems. Several avenues of future research can be pursued to improve the method. Two of the most promising areas of research have been identified. The first is the investigation on how uncertainties in the model affect the layout of architectures. The current model assumes deterministic information, for instance in model input parameters. Uncertainty plays a significant role since decisions are made based on assumptions (such as, for example, market cost of construction in the offshore oil field application case) that might change over time. The second area of future research of interest is to study how distributed decision-making structures affect the architecting process, relaxing the assumption in this paper that assumed centralized design authority on the system architecture.

\section{ACKNOWLEDGMENTS}

This work has been sponsored by the BP-MIT Major Projects Research Program. The authors acknowledge technical support and guidance from Dr. Bob Robinson, Dr. Rene Keller, and Dr. Tomas Flanagan from the BP Concept Modeling and Development Group. The authors also thank the reviewers for comments and suggestions for improvement of the paper.

\section{REFERENCES}

J. Agte, O.L. de Weck, J. Sobieszczanski-Sobieski, P. Arendsen, A. Morris, and M. Spieck, MDO: Assessment and direction for advancement-an opinion of one international group, Struct Multidisciplinary Optim J 40 (2010), 17-33.

J.S. Aronofsky and A.C. Williams, The use of linear programming and mathematical models in underground oil production, Management Sci 8(4) (1962), 394-407. 
R.J. Barnes and A. Kokossis, Optimal platform design of offshore fields with satellite production, Comput Aided Chem Eng 20 (2005), 679-684.

A.C. Bittencourt, Optimal scheduling of development in an oil field, MS Report, Stanford University, Palo Alto, CA, 1994.

J. Bohannon, A linear programming model for optimum development of multi-reservoir pipeline systems, J Pet Technol 22(11) (1970), 1429-1436.

M.D. Devine and W.G. Lesso, Models for the minimum cost development of offshore oil fields, Management Sci 18(8) (1972), 378-387.

J.S. Dyer, P.C. Fishburn, R.E. Steuer, J. Wallenius, and S. Zionts, Multiple criteria decision making, multiattribute utility theory: The next ten years, Management Sci 38(5) (1992), 645-654.

W.K. Hofstetter, A framework for the architecting of aerospace systems portfolios with commonality, Ph.D. dissertation, Massachusetts Institute of Technology, Cambridge, MA, 2009.

R.R. Iyer, I.E. Grossmann, S. Vasantharajan, and A.S. Cullick, Optimal planning and scheduling of offshore oil field infrastructure investment and operations, Indust Eng Chem Res 37 (1998), 1380-1397.

R. Keeney and H. Raiffa, Decisions with multiple objectives, Wiley, New York, 1976.

H.Y.B. Koo, A meta-language for systems architecting, Ph.D. dissertation, Massachusetts Institute of Technology, Cambridge, MA, 2005.

W.L. Leffler, R. Pattarozzi, and G. Sterling, Deepwater petroleum exploration \& production-a nontechnical guide, PennWell Books, Tulsa, OK, 2003.

J. Lin, Exploring flexible strategies in engineering systems using screening models - applications to offshore petroleum projects,
Ph.D. dissertation, Massachusetts Institute of Technology, Cambridge, MA, 2008.

X. Lin and C.A. Floudas, A novel continuous-time modeling and optimization framework for well platform planning problems, Optim Eng 4(1) (2003), 65-95.

M. Maier, Architecting principles for systems-of-systems, Syst Eng 1(4) (1998), 267-284.

M. Maier and E. Rechtin, The art of systems architecting, 2nd edition, CRC Press, Boca Raton, FL, 2002.

B. Prasad, Concurrent engineering fundamentals- integrated product and process organization, Prentice Hall, Upper Saddle River, NJ, 1996.

M.S. Raymond and W.L. Leffler, Oil and gas production in nontechnical language, PennWell Books, Tulsa, OK, 2006.

M. Saravi, L. Newnes, A.R. Mileham, and Y.M. Goh, "Estimating cost at the conceptual design stage to optimize design in terms of performance and cost," Collaborative Product and service life cycle management for a sustainable world, R. Curran, S.Y. Chou, and A. Trappey (Editors), Springer, London, 2008.

W.L. Simmons, A framework for decision support in systems architecting, Ph.D. dissertation, Massachusetts Institute of Technology, Cambridge, MA, 2008.

R. Starr, Optimal production and allocation under uncertainty, Quart J Econ 87(1) (1973), 81-95.

S.A. van den Heever and I. E. Grossmann., An iterative aggregation/disaggregation approach for the solution of a mixed-integer nonlinear oilfield infrastructure planning model, . Indust Eng Chem Res 39(6) (2000), 1955-1971.

M. Zeleny, Multiple criteria decision making, McGraw-Hill, New York, 1982.

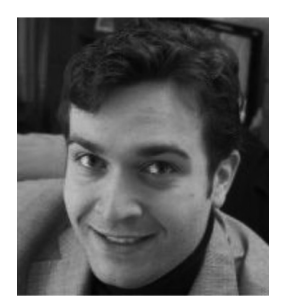

Alessandro Aliakbargolkar is Assistant Professor at Skolkovo Institute of Science and Technology in Moscow, Russian Federation. He received a Ph.D. in Aeronautics and Astronautics from MIT in 2012. His research interests lie in the areas of systems architecture, project management, systems engineering, and spacecraft design analysis and optimization. He is a Certified INCOSE Associate Systems Engineering Professional, and a registered Industrial Engineer in Italy, with research experience at Caltech/NASA Jet Propulsion Laboratory, and at the European Space Agency. Before MIT, Alessandro received a Laurea degree in Aerospace Engineering in 2006 and a Laurea Specialistica degree in Astronautics Engineering in 2008 from University of Rome "La Sapienza," Italy.

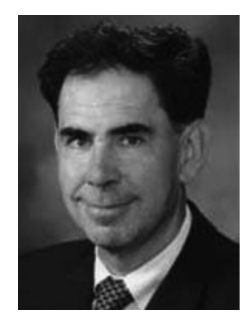

Edward Crawley received an Sc.D. in Aerospace Structures from MIT in 1981. His early research interests centered on structural dynamics, aeroelasticity, and the development of actively controlled and intelligent structures. Recently, Dr. Crawley's research has focused on the domain of the architecture and design of complex systems. From 2003 to 2006 he served as the Executive Director of the Cambridge-MIT Institute. For the previous 7 years, he served as the Department Head of Aeronautics and Astronautics at MIT, leading the strategic realignment of the department. Dr. Crawley is a Fellow of the AIAA and the Royal Aeronautical Society (UK), and is a member of three national academies of engineering: the Royal Swedish Academy of Engineering Science, the (UK) Royal Academy of Engineering, and the US National Academy of Engineering. He is the author of numerous journal publications in the AIAA Journal, the ASME Journal, the Journal of Composite Materials, and Acta Astronautica. In his outreach and public service, Dr. Crawley was chairman of the NASA Technology and Commercialization Advisory Committee, and was a member of the NASA Advisory Committee. He received the NASA Public Service Medal. In 1993, he was a member of the Presidential Advisory Committee on the Space Station Redesign. He is conversant in Russian, and has spent time as a visitor at the Moscow Aviation Institute, the Beijing University of Aeronautics and Astronautics, Stanford University, Palo Alto, CA, and Cambridge University. He was a finalist in the NASA Astronaut selection in 1980, is an active pilot, and was the 1990, 1995, and 2005 Northeast Regional Soaring champion. In 2004 he received the Distinguished Eagle Scout Award of the Boy Scouts of America. Recently, Professor Crawley was one of the 10 members of the presidential committee led by Norman Augustine to study the future of human spaceflight in the US. 